\title{
ANALYSIS OF LOAD BALANCING ALGORITHMS IN CLOUD COMPUTING
}

\author{
Chitranshi Gautam \\ B.Tech. Scholar, \\ Department of CSE \\ Arya Institute of Engineering \\ and Technology, Kukas, \\ Jaipur, Rajasthan
}

\author{
Dikshita Singh \\ B.Tech. Scholar, \\ Department of CSE \\ Arya Institute of Engineering \\ and Technology, Kukas, \\ Jaipur, Rajasthan
}

\author{
Arpit Sharma \\ B.Tech. Scholar, \\ Department of CSE \\ Arya Institute of Engineering \\ and Technology, Kukas, \\ Jaipur, Rajasthan
}

\begin{abstract}
Cloud provides the benefits of computing resources be it software, platform or infrastructure. These services are based on user's demands which are shared over a virtualized environment. When the user's request goes high it becomes difficult to manage these requests at one time. Thus there is a necessity to distribute this workload and computing resources fairly across one or more servers. A load balancer is like a "traffic cop" which directs the traffic to avoid a situation where few servers are overloaded while few are idle. Proper load balancing can ensure maximum throughput in minimum response time. This paper is a critical review of all the existing techniques of load balancing and comparison between them.
\end{abstract}

Keywords - cloud computing, load balancing, load balancing algorithms (static and dynamic), smart load balancing.

\section{INTRODUCTION}

Owing to the advantages like cost efficiency, ease of access and almost unlimited storage, cloud computing has emerged as the fastest growing technology in the IT industry. Cloud computing provides infrastructure as a service(IAAS), platform as a service(PAAS) and software as a service(SAAS), these services are available to consumers under the pay as you go basis. Every other person wants to use these services as it reduces the cost of hardware, provides 24/7 access from any corner of the world and also ensures data security.

A cloud consists of several computers or servers (nodes) connected to form a cluster. When too many random requests are generated by clients, it is apparent that some of these servers may get overloaded. This overloading of servers can deteriorate the performance of our cloud. Load imbalance may cause system bottleneck that is when workloads arrive too quickly for the processors to handle. The ineffectiveness brought about by the bottleneck often creates delays. These conditions are raising a high demand of load balancers or effective load balancing techniques. Effective load balancing results in minimizing the downtime, implementing fail-overs, enabling scalability, avoiding bottlenecks and overprovisioning.

The servers are legitimately assembled into clusters and the task of load balancing is disseminated among these clusters or groups. The load is allocated to the servers present in that cluster. Servers belonging to a cluster offer same services. Various load balancing approaches have been actualized for the cloud condition to provide productive load distribution.

This paper is requested as follows. Area II depicts what is load balancing and a portion of its estimating parameters. Area III depicts the classification of load balancing algorithms. In segment IV comparison among various load balancing calculation is given.

\section{LOAD BALANCING}

Cloud load balancing is a technique for parting outstanding burdens progressively and consistently over various application servers running on cloud foundation. It would have been very difficult to manage the cloud technology not having the benefit of load balancing. It provides necessary redundancy techniques to make unreliable system reliable via managed redirection. Load balancing system can use a different mechanism to assign directions to various services. Simply load balancers are used to listen to network ports of the service request. Whenever we face mounting demand from users, load-balancers use the scheduling algorithm to assign where the request is sent.

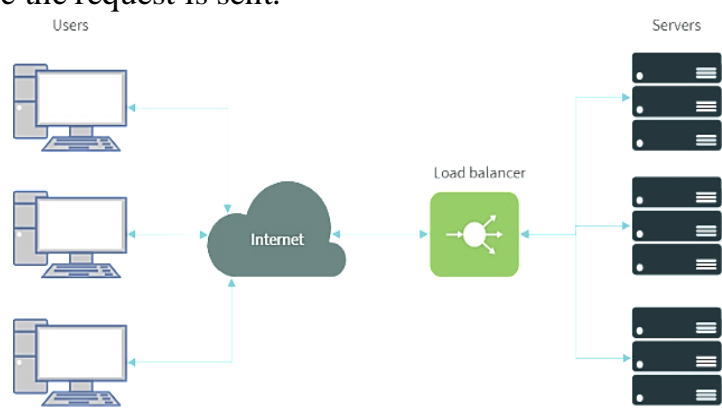

Fig1 - Load Balancing in Cloud Computing 


\section{International Journal of Engineering Applied Sciences and Technology, 2020 \\ Vol. 4, Issue 12, ISSN No. 2455-2143, Pages 122-126 \\ Published Online April 2020 in IJEAST (http://www.ijeast.com)}

Load balancing techniques can be evaluated based on some parameters Kanakala R. et al. $(2015,2017)$ in their work have mentioned some parameters like throughput, response time, performance, scalability, resource utilization and fault tolerance.

- Throughput - In terms of the cloud is the amount of data that can be transferred from one location to another in a given amount of time.

- Response Time - It is the time interim between a client solicitation and receipt of activity or feedback from the system.

- Performance - It is a measure of accuracy, cost and speed of the working algorithm.

- Scalability - It is the adaptability of the algorithm according to the required conditions.

- Resource Utilization - It is the effective use of resources as per requirement.

- Fault Tolerance - It is the property that enables a system to continue to work properly on the occurrence of failure.

A good load balancing algorithm aims to:

1. To maximize throughput.

2. To reduce response time.

3. To optimize resource utilization.

4. To avoid overload of any single resource.

\section{CLASSIFICATION OF LOAD BALANCING ALGORITHMS}

There are a few Load Balancing algorithms which are extensively characterized into two classifications dependent on framework load:-

1. Static Load Balancing

2. Dynamic Load Balancing

Static Load Balancing: It is an approach where load balancing is achieved by providing prior information about the system. The performance of the node is determined at the beginning of execution. Nodes calculate the work allotted to them and submit the result to the remote node. Then based on performance workload is distributed in start without considering the current load. Static load balancing methods are non-preemptive i.e when the load is assigned to one node it cannot be moved to another node. This fundamental bit of leeway of this methodology is that it requires less correspondence thus diminishes the execution time the principle disadvantage of this methodology is that it doesn't think about the present condition of the system while settling on allocation choices.

Some static load balancing algorithms are round robin, minmin, max-min and randomized load balancing algorithm.

\section{A. ROUND ROBIN ALGORITHM}

It is a load balancing technique where a DNS server rotates which out of several servers IP addresses is to be used. It has a list of IP addresses and provides a different IP address for each successive request, returning to the first one after the last has been provided. The main advantage of using round robin load balancing is its simple implementation. But it does not always result in the most efficient traffic distribution, because many round robin load balancers assume that all servers are the same.

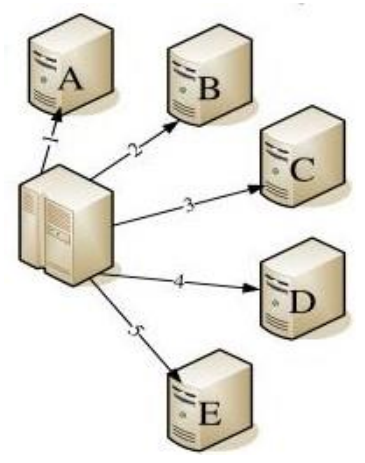

Fig2 - Round Robin Load Balancing Technique

\section{B. MIN-MIN ALGORITHM}

In min-min load balancing a task having minimum completion time (MCT) is assigned first for execution. As proposed in references [3], [12] this algorithm is implemented into two steps: In the first step, the expected completion time for each task in metatask is calculated on each resource. In the second step, the task with minimum expected completion time is selected and assigned to the corresponding resource, after that the selected task is removed from metatask. This process is repeated until all the tasks in metatask get mapped. This algorithm is simple to implement if the size of the task in metatask is small, if there are large size tasks then it gives poor resource utilization as large size tasks have to wait for smaller tasks to complete.

\section{MAX-MIN ALGORITHM}

The working of the max-min algorithm [11] is similar to the min-min algorithm the only difference is that in max-min algorithm priority is given to the task with highest completion time (HCT). Once the lengthy tasks are completed, the task with minimum execution time is assigned to the processor. This algorithm also maintains time sequences of the task engaged and keep informed the execution time periodically to the load balancers as well as processor. The disadvantage of this algorithm is that it might increase the total response time of the system due to the execution of the task with HCT first thus making it inefficient. 


\section{RANDOMIZED ALGORITHM}

It is another approach to load balancing in this a list of server IPs is delivered to the client, and then the client can randomly select the IP from the list. The client-side random load balancing tends to provide better distribution of load as compared to round robin.

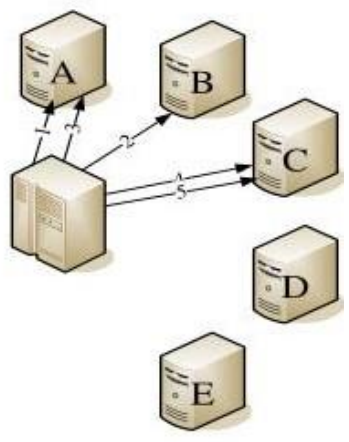

Fig3 - Randomized Load Balancing Technique

Dynamic Load Balancing: These algorithms watch changes on the system outstanding task at hand and redistribute the work as needs be. Dynamic algorithms can have three distinctive controlling structures: concentrated, distributed, or semi-distributed. In brought together load circulation, a solitary node (focal hub) is liable for all load dispersion in the system. In appropriated the duty is similarly isolated among all nodes. Though in a semi-distributed the system is portioned into groups where each group is centralized.

This algorithm comprises of three strategies: transfer strategy, location strategy and information strategy.

- Transfer strategy - chooses which tasks are qualified for move to different nodes for handling.

- Location strategy - proposes a remote node to execute a transferred task.

- Information strategy - is the data place for the load balancing algorithm. It provides area and transfer strategies to each node.

Some dynamic load balancing algorithms are ESCEA, throttled, biased random, token rating, honey bee foraging behaviour and ANT COLONY load balancing algorithm.

\section{A. ESCE (Equally Spread Current ALGORITHM}

Execution)

ESCE is also called as active VM (Virtual Machine) load balancing algorithm. A.A. jaiswal, et al. (2014) and surbhi kapoor, et al. (2015) have demonstrated that this algorithm equally distributes the workload on each VM. It maintains a list of virtual machines and the number of requests already allotted to that virtual machine. When a new client request is received DCC (Data Centre Controller) asks ESCE load balancer for next VM allocation. It will then scan the list from starting to get a least loaded VM. If there is more than one virtual machine present then the first one is chosen and its id is sent to DCC. DCC allots the client request to the selected VM and the list is updated by an increasing number of allocation counts of that particular VM. The main disadvantage of ESCE load balancing is its high computational overhead.

\section{B. THROTTLED ALGORITHM}

This load balancing algorithm is generally appropriate for virtual machines. In this load balancer keeps up the list of entire virtual machines in the system and their states whether they are accessible or occupied (busy). On getting a customer demand, it checks the ordering table. If the VM is accessible, at that point the task is appointed to that machine. If the fitting VM isn't discovered, at that point it restores a worth 1 to DCC and places the request in the line. After finishing the processing of the designated demand, reaction cloudlets are sent to DCC which consequently send a notice for deallotment. The ordering table is refreshed after every assignment and de-allotment of the resource. This algorithm has better resource use yet checks the whole list from the earliest starting point.

\section{BIASED RANDOM ALGORITHM}

Biased random algorithm adjusts the load through random sampling of framework area. In this virtual chart of the framework is made. In a directed graph, every node signifies a vertex and each in-degree signifies free resources of that node. The load is distributed by the load balancer to the node which has at any rate one in-degree. The in-level of the node is increased and decremented when the undertaking is finished and when the assignment is designated separately. This is done through the procedure of random sampling. This algorithm is appropriate for huge networks.

\section{HONEY BEE FORAGING BEHAVIOUR ALGORITHM}

This algorithm is based on the behaviour of real honey bees in finding their food sources. There are three groups of bees: employed bees, onlookers and scouts. Every employed bee goes to a food source to determine a neighbouring source, then evaluates its nectar amount and dances in the hive. Each onlooker chooses one of the sources depending on the dances of the employed bees and then goes to that source. The bee evaluates the nectar amount after choosing a neighbour around that source. The abandoned food sources are discovered and are replaced with the new food sources discovered by scouts. The best food source found is registered. The disadvantage of this algorithm is that throughput will not increase with the increase in resources. 


\section{E. ANT COLONY ALGORITHM}

We have gone through the work of A.A. jaiswal et al. (2014) and Sarmila G.Punetha, et al. (2015) to get that Ant Colony Algorithm is a nature inspired algorithm, this algorithm depends on the conduct of genuine ants. In this, the ant picks the shortest path in search of its food. At the point when the request is initiated ant starts its movement. Ant will ceaselessly check whether the node is overloaded or under loaded. In the event that an ant finds an over-burden node, it will turn around. Also, if an ant finds any under loaded node, it will continue. Along these lines, ant's conduct is utilized to gather data from various nodes. The disadvantage of this algorithm is that delay can be caused by moving forward and backwards.

\section{COMPARISON AMONG DIFFERENT LOAD BALANCING ALGORITHMS}

\begin{tabular}{|l|l|}
\hline Algorithm & Advantage \& Disadvantage \\
\hline $\begin{array}{l}\text { Round Robin } \\
\text { Algorithm }\end{array}$ & $\begin{array}{l}\text { Simple, fast response, no starvation but } \\
\text { not scalable. }\end{array}$ \\
\hline $\begin{array}{l}\text { Min-Min } \\
\text { Algorithm }\end{array}$ & $\begin{array}{l}\text { Simple and fast for smaller tasks but cause } \\
\text { starvation of larger tasks. }\end{array}$ \\
\hline $\begin{array}{l}\text { Max-Min } \\
\text { Algorithm }\end{array}$ & $\begin{array}{l}\text { Simple in execution but cause starvation } \\
\text { of smaller tasks. }\end{array}$ \\
\hline ESCE Algorithm & $\begin{array}{l}\text { Equal load balancing, maximize } \\
\text { throughput but not fault tolerant. }\end{array}$ \\
\hline $\begin{array}{l}\text { Throttled } \\
\text { Algorithm }\end{array}$ & $\begin{array}{l}\text { Good performance, better resource } \\
\text { utilization but scans the entire list from the } \\
\text { beginning. }\end{array}$ \\
\hline $\begin{array}{l}\text { Biased Random } \\
\text { Algorithm }\end{array}$ & $\begin{array}{l}\text { Suitable for large networks but } \\
\text { performance degrades with an increase in } \\
\text { diversity. }\end{array}$ \\
\hline $\begin{array}{l}\text { Honeybee } \\
\text { Foraging } \\
\text { Behaviour } \\
\text { Algorithm }\end{array}$ & $\begin{array}{l}\text { Performance increases by increasing } \\
\text { system size, throughput will not increase } \\
\text { with the increase in resources. }\end{array}$ \\
\hline $\begin{array}{l}\text { Ant Colony } \\
\text { Algorithm }\end{array}$ & $\begin{array}{l}\text { Decentralized, has network overhead, } \\
\text { good resource utilization but causes delay. }\end{array}$ \\
\hline
\end{tabular}

\section{CONCLUSION}

In this paper, we have studied about load balancing and how different static and dynamic load balancing algorithms work in equally distributing the load. These classic load balancing algorithms play an essential role but there is a need to evolve. So it would be better if load balancers are designed smart enough (smart load balancers) to recognize technical characteristics like response time, execution time, size of data and load on each resource. Load balancers can be made to learn through their experiences to recognize an incoming query and respond accordingly.

\section{ACKNOWELEGMENT}

Under the guidance of Mr. Indra Kishor, Associate Prof. Department of CSE, Arya Institute of Engineering and Technology, we have created a paper on Analysis of Load Balancing Algorithms in Cloud Computing.

\section{REFRENCES}

[1] Kandi R.; Anand A., 2018, "Analytical Study of different Load balancing algorithms", International Jornal of Advanced Studies in Computer Science and Engineering IJ ASCSE Volume 7, Issue 1.

[2] Sajjan R.S; Yashwantrao B.R., 2017, "Load Balancing and its Algorithms in Cloud Computing: A Survey", International Journal of Computer Sciences and Engineering Volume 5(1).

[3] Nehasharma, Dr.Sanjaytyagi, March-April2017, "Compartive analysis of min-min and max-min on the basis of makespan parameters", International Journal of Advanced Research in Computer Science,8(3), 1038-104.

[4] Khanchi M. and Tyagi S., 2016, 'An Efficient Algorithm for Load Balancing in cloud computing', International Journal of Engineering Sciences \& Resarch Technology, ISSSN: 2277-9655.

[5] Kanakala R.; Reddy V.K.; Karthik K., 2015, "Performance analysis of load balancing techniques in cloud computing environment", International Journal of Computer Sciences and Engineering Vol.-5(1), Jan 2017, E-ISSN: 2347-2693.

[6] Aslam S., Shah M.A., 2015, "Load Balancing Algorithms" in Software Engineering Conference (NSEC).

[7] Kumar R. and Prashar T., 2015, 'Performance Analysis of Load Balancing Algorithms in cloud computing', International journal of computer Applications, Vol.120, No.7, pp 19-27. 
[8] Kapoor S., Dr. Chetna Dabas, 2015, "Cluster Based Load Balancing in Cloud Computing", Eighth International Conference on Contemporary Computing (IC3).

[9] Sarmila G.Punetha, Dr.N.Gnanambigai, Dr.P.Dinadayalan, 2015, "Survey on Fault Tolerant Load Balancing Algorithms in Cloud Computing", IEEE Sponsored 2nd International Conference On Electronics And Communication System (ICECS), Pages1715-1720.

[10] Jaiswal A.A., Dr. Sanjeev Jain, 2014, “ An Approach towards the Dynamic Load Management Techniques in Cloud Computing Environment", International Conference on Power, Automation and Communication (INPAC).

[11] Santhosh, B., \& Manjaiah, D. (2014),"An improved task scheduling algorithm based on max-min for cloud computing",International conference on Advances in computer \& communications Engineering(ACCE2014),vol 2,issue2,may2014.

[12] Yu, Xiaogao, and Xiaopeng Yu., 2009, "A new grid computation-based Min-Min algorithm", Fuzzy Systems and Knowledge Discovery, FSKD'09 Sixth International Conference on, Volume 1, Pages $43-$ 45, IEEE.

[13] Report TR06, 2005, Computer Engineering Department, Erciyes University, Turkey. 